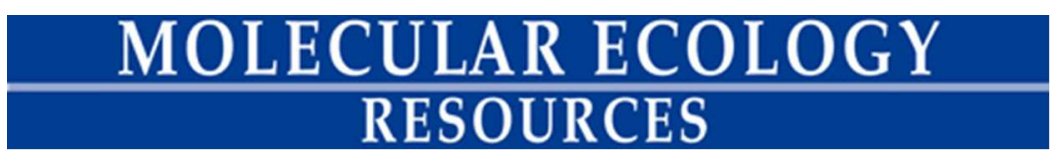

\title{
Combining bleach and mild pre-digestion improves ancient DNA recovery from bones
}

\begin{tabular}{|r|l|}
\hline Journal: & Molecular Ecology Resources \\
\hline Manuscript ID & MER-16-0205.R1 \\
\hline Manuscript Type: & Resource Article \\
\hline Date Submitted by the Author: & n/a \\
\hline Complete List of Authors: & $\begin{array}{l}\text { Boessenkool, Sanne; Universitetet i Oslo Det Matematisk- } \\
\text { naturvitenskapelige Fakultet } \\
\text { Hanghøj, Kristian; Kobenhavns Universitet Statens Naturhistoriske } \\
\text { Museum; Universite Toulouse III Paul Sabatier } \\
\text { Nistelberger, Heidi; Universitetet i Oslo Det Matematisk- } \\
\text { naturvitenskapelige Fakultet } \\
\text { Der Sarkissian, Clio; University of Copenhagen, Centre for Geogenetics } \\
\text { Gondek, Agata; Universitetet i Oslo Det Matematisk-naturvitenskapelige } \\
\text { Fakultet } \\
\text { Orlando, Ludovic } \\
\text { Barrett, James; University of Cambridge } \\
\text { Star, Bastiaan; Universitetet i Oslo Det Matematisk-naturvitenskapelige } \\
\text { Fakultet }\end{array}$ \\
\hline Keywords: & $\begin{array}{l}\text { ancient DNA, DNA extraction, contamination, high-throughput sequencing, } \\
\text { Atlantic cod, horse }\end{array}$ \\
\hline \hline & \\
\hline
\end{tabular}

\section{SCHOLARONE ${ }^{\text {m }}$}


1 Combining bleach and mild pre-digestion improves ancient DNA recovery from bones

2 Sanne Boessenkool ${ }^{1}$, Kristian Hanghøj ${ }^{2,3}$, Heidi M. Nistelberger ${ }^{1}$, Clio Der Sarkissian ${ }^{2}$, Agata

3 Gondek $^{1}$, Ludovic Orlando ${ }^{2,3}$, James H. Barrett ${ }^{4}$, Bastiaan $\operatorname{Star}^{1}$

4

$5{ }^{1}$ Centre for Ecological and Evolutionary Synthesis, Department of Biosciences, University of 6 Oslo, PO Box 1066, Blindern, N-0316 Oslo, Norway.

$7{ }^{2}$ Centre for Geogenetics, Natural History Museum of Denmark, University of Copenhagen, 8 Øster Voldgade 5-7, 1350 Copenhagen K, Denmark.

$9 \quad{ }^{3}$ Université de Toulouse, University Paul Sabatier (UPS), Laboratoire AMIS, CNRS UMR 105288 , Toulouse, France

$11{ }^{4}$ Division of Archaeology, University of Cambridge, Downing Street, Cambridge CB2 3DZ, 12 United Kingdom.

14 Keywords:

15 Ancient DNA, DNA extraction, contamination, high-throughput sequencing, Atlantic cod, 16 horse

17 Corresponding authors:

18 Sanne Boessenkool, Centre for Ecological and Evolutionary Synthesis, Department of 19 Biosciences, University of Oslo, PO Box 1066, Blindern, N-0316 Oslo, Norway. 20 sanne.boessenkool@ibv.uio.no

21 Bastiaan Star, Centre for Ecological and Evolutionary Synthesis, Department of Biosciences, 22 University of Oslo, PO Box 1066, Blindern, N-0316 Oslo, Norway. bastiaan.star@ibv.uio.no 23

24 Running title: Improved access to ancient DNA 


\section{Abstract}

26 The feasibility of genome-scale studies from archaeological material remains critically 27 dependent on the ability to access endogenous, authentic DNA. In the majority of cases, this 28 represents a few percent of the DNA extract, at most. A number of specific pre-extraction 29 protocols for bone powder aimed to improve ancient DNA recovery before library 30 amplification have recently been developed. Here, we test the effects of combining two of 31 such protocols, a bleach wash and a pre-digestion step, on 12 bone samples of Atlantic cod 32 and domestic horse aged 750-1350 cal. years before present. Using high-throughput 33 sequencing, we show that combined together, bleach wash and pre-digestion consistently 34 yield DNA libraries with higher endogenous content than either of these methods alone. 35 Additionally, the molecular complexity of these libraries is improved and endogenous DNA 36 templates show larger size distributions. Other library characteristics, such as DNA damage 37 profiles or the composition of microbial communities are little affected by the pre-extraction 38 protocols. Application of the combined protocol presented in this study will facilitate the 39 genetic analysis of an increasing number of ancient remains and will reduce the cost of whole 40 genome sequencing. 


\section{Introduction}

Archaeological bone material represents the primary source of ancient DNA (aDNA) (e.g. Hofreiter, et al. 2014; Der Sarkissian, et al. 2015). High-throughput DNA sequencing of aDNA extracts typically allows for the recovery of portions of the nuclear and mitochondrial genomes. The practical and financial feasibility of such studies is critically dependent on our ability to retrieve the degraded DNA molecules that survived after death. While petrosal bones represent exceptions (e.g. Reich, et al. 2010; Meyer, et al. 2012; Gamba, et al. 2014; Lazaridis, et al. 2014; Pinhasi, et al. 2015), the fraction of endogenous DNA present in bones, also referred to as their endogenous content, is typically low and in the range of a few percent at most (Carpenter, et al. 2013). Consequently, a major challenge of working with ancient bone material is maximizing cost-efficiency when using high-throughput sequencing at genome-wide scales (Rizzi, et al. 2012).

In recent years, continuing attention has been paid to developing novel experimental methods to improve access to the endogenous content from ancient samples. For instance, hybridization capture techniques aim to enrich entire genomes or specific sections after library preparation by annealing templates to pre-selected DNA baits (Briggs, et al. 2009; Carpenter, et al. 2013; Enk, et al. 2014). Other approaches improve access to the endogenous DNA fraction at the extraction stage, which minimizes ascertainment bias and other experimental bias associated with capture based methods (Castellano, et al. 2014). As such, the surface of bone samples is typically cleaned by mechanical removal of the outer layer, and the application of chemical and/or enzymatic treatments (Kemp and Smith 2005; Malmström, et al. 2007; Li and Liriano 2011). More recently, methods to clean bone powder - i.e. after homogenization of the bone sample - have been shown to significantly increase the fraction of endogenous templates sequenced, especially through the washing of bone powder with 
bleach or phosphate (Korlevic, et al. 2015), or a proteinase K “pre-digestion" step prior to DNA extraction (Orlando, et al. 2011; Ginolhac, et al. 2012; Der Sarkissian, et al. 2014; Gamba, et al. 2014; Damgaard, et al. 2015; Gamba, et al. 2016). The success of these methods indicates that endogenous DNA is more tightly bound to the bone matrix than exogenous DNA, perhaps in micro-niches in the bone material (Campos, et al. 2012; Ginolhac, et al. 2012) and provides a rationale to further investigate simple, cost-effective washing methods for the pre-treatment of ancient bone material.

Here, we apply high-throughput sequencing to investigate the efficiency of a combined bleach wash and pre-digestion on the endogenous content of 12 ancient bone samples from Atlantic cod (Gadus morhua) and horse (Equus caballus), dated to 750-1350 cal. years before present (BP) and coming from various archaeological contexts. We show that combined together, bleach wash and pre-digestion consistently increase the endogenous DNA content. This procedure leads to a 1.7 to 3 -fold increase relative to the pre-digestion protocol in absence of a bleach wash. This improvement is not associated with a significant increase in DNA damage patterns or changes in the composition of microbial communities. Our results indicate that a multiplicative effect enriches endogenous DNA prior to library build, and highlight the effectiveness of treating bone powder prior to DNA extraction.

\section{Material and methods}

\section{Sample information}

Bones from Atlantic cod (Gadus morhua) and horse (Equus caballus) were obtained from Quoygrew and the Brough of Deerness, Orkney, Scotland, and the Gokstad ship burial, Norway (Table S1, Supporting Information; Bonde and Christensen 1993; Barrett and Slater 2009; Barrett 2012). Age estimates (between 1350 and 750 cal. years BP) were indirectly 
90 obtained based on associated archaeological remains. We ensured that bones from

91 independent individuals were collected, by using the same bone type within a stratum or 92 bones from different strata within the archaeological site.

Sampling and DNA extraction

All laboratory work preceding PCR was done in the dedicated ancient DNA laboratories at 96 the Institute of Biosciences, University of Oslo following strict DNA precautions (Poinar and 97 Cooper 2000; Gilbert, et al. 2005). Bone samples were exposed to UV for 10 minutes on each side resulting in a total dosage of $4800 \mathrm{~J} / \mathrm{m} 2$ before being cut and milled to powder. Atlantic cod bones were milled completely in a Retsch MM400, while horse bones or teeth were subsampled (cutting out a part of the root) using a scalpel attached to a Perfecta 600 (W\&H).

101 The surface of each subsample was removed using a drill bit. All subsamples were

102 subsequently exposed to UV (describe above) before being homogenised in a Retsch MM400

103 mixer mill. Washes and DNA extractions were performed on $200 \mathrm{mg}$ of homogenized bone 104 powder unless stated otherwise.

107 DNA was extracted from powder from five Atlantic cod samples and seven horse samples

108 after two different treatments: 1) a double digestion, whereby the bone powder remaining 109 after a first, short digestion or pre-digestion step was subjected to a second digestion using a 110 fresh digestion buffer (DD), and 2) a bleach wash followed by a double digestion (BleDD);

111 Fig. 1). For the cod samples, we also extracted DNA from the supernatant of the first, short 112 digestion, both when bone powder was subjected only to digestion directly after 113 homogenization (short digestion, SD) and when bone powder was washed with bleach prior 
114 to the short digestion step (BleSD). Finally, we tested the effect of adding twice the amount

115 (i.e. two times $200 \mathrm{mg}$ ) of starting material for Atlantic cod bones when using BleDD 116 (BleDD ${ }^{2}$; Fig. 1).

117 Washing of bone powder with bleach was carried out by incubating 150-200 mg bone 118 powder in $1 \mathrm{ml}$ of $0.5 \%$ bleach solution (VWR) in $2 \mathrm{ml}$ tubes for $15 \mathrm{~min}$ at room temperature 119 (RT) under gentle 3-dimensional shaking using a nutating mixer. After incubation, bleach was 120 removed by centrifugation and removal of supernatant and washing of bone powder 121 repetitively (three times) with $1 \mathrm{ml} \mathrm{UV-treated} \mathrm{milli-Q} \mathrm{water,} \mathrm{whereby} \mathrm{thorough} \mathrm{contact}$ 122 between water and bone powder was ensured by vortexing before centrifugation, as per 123 Korlevic et al. (2015). For BleDD ${ }^{2}$, bleach wash and digestion with $200 \mathrm{mg}$ of bone powder 124 were performed in two separate tubes and combined following the second digestion (see 125 below).

126 The short, pre-digestion treatment of samples following Damgaard, et al. (2015) was 127 carried out in $1 \mathrm{ml}$ volumes containing $0.45 \mathrm{M}$ EDTA $(\mathrm{pH} 8.0), 25$ ųl proteinase $\mathrm{K}(\sim 18$ $128 \mathrm{mg} / \mathrm{ml}$; Roche) and $0.5 \% \mathrm{~N}$-Laurylsarcosyl. Incubations were performed at $37^{\circ} \mathrm{C}$ under gentle 129 3-dimensional shaking for one hour. Following centrifugation, the supernatant was directly 130 transferred to a new tube for extraction of DNA (SD and BleSD protocols) or stored at $-20^{\circ} \mathrm{C}$ 131 for later extraction. Following the removal of the supernatant from this first, short digestion, 132 the remaining bone powder was subjected to an overnight, second digestion using a freshly 133 prepared digestion buffer (DD, BleDD and BleDD ${ }^{2}$ protocols). Following Gamba, et al. 134 (2016), in all protocols the supernatant was concentrated to 200 ull by adding it to $2 \mathrm{ml} 10 \mathrm{mM}$ 135 Tris-EDTA (Sigma-Aldrich) and spinning through an Amicon Ultra 4 (30 kD). DNA was 136 subsequently extracted using Qiagen Minelute columns according to manufacturer's 
137 instructions, and eluted in 60 ųl pre-heated $\left(60^{\circ} \mathrm{C}\right) \mathrm{EB}$ buffer after a 10 minute incubation at

$13837^{\circ} \mathrm{C}$. Negative controls were included in all extraction experiments.

Library preparation and sequencing

141 Blunt-end Illumina libraries as described by Meyer and Kircher (2010) were built following

142 Schroeder, et al. (2015) with the exception that all reactions were performed in half volumes.

143 Sample-specific seven bp indexes in the P7 primer were used to allow multiplexing of

144 libraries for sequencing. Library amplification was performed in $15 \mu \mathrm{l}$ of ligated DNA with

1452.5 U PfuTurbo Cx Hotstart DNA Polymerase (Agilent Technologies), 1x buffer, $0.2 \mathrm{mM}$ per

$146 \mathrm{dNTP}, 0.2 \mu \mathrm{M}$ P7 index primer, $0.2 \mu \mathrm{M}$ P5 IS4 primer and $0.4 \mathrm{mg} / \mathrm{ml}$ BSA. Reactions were

147 amplified for 13 cycles $\left(2 \min\right.$ at $95^{\circ} \mathrm{C}, 13$ cycles of $30 \mathrm{~s}$ at $95^{\circ} \mathrm{C}, 30 \mathrm{~s}$ at $60^{\circ} \mathrm{C}$ and $70 \mathrm{~s}$ at $72^{\circ} \mathrm{C}$

148 with a final extension of $10 \mathrm{~min}$ at $72^{\circ} \mathrm{C}$ ). Amplified library products were subsequently

149 cleaned using Agencourt ${ }^{\circledR}$ AMPure XP beads at a 1:1.7 ratio (PCR volume: AMPure

150 volume), eluted in $30 \mu 1$ sterile Millipore water and visualised and quantified on a Bioanalyzer

1512100 (Agilent). Subsets of libraries that required further amplification due to low

152 concentration were split over four separate reactions to minimize library clonality. These re-

153 amplifications were performed using $1 \mathrm{U}$ Accuprime Pfx DNA polymerase (Invitrogen), 1x

154 buffer, $0.25 \mu \mathrm{M}$ IS5, $0.25 \mu \mathrm{M}$ IS6 and 1 unit polymerase) and amplified with the following

155 PCR profile: $2 \mathrm{~min}$ at $95^{\circ} \mathrm{C}, 4$ cycles of $20 \mathrm{~s}$ at $95^{\circ} \mathrm{C}, 20 \mathrm{~s}$ at $60^{\circ} \mathrm{C}$ and $40 \mathrm{~s}$ at $72^{\circ} \mathrm{C}$, final

156 extension step of $3 \mathrm{~min}$ at $72^{\circ} \mathrm{C}$ (see Tables 1 and 2 for the total number of cycles each

157 library was amplified for). Re-amplified libraries were pooled before cleanup with AMPure as

158 described above. Sequencing of libraries was carried out on an Illumina Hiseq 2500 at the

159 Norwegian Sequencing Centre (125 bp paired-end) and data were demultiplexed following

160 standard procedure allowing no mismatches in the index sequences. To monitor 
161 contamination libraries were also built of DNA extraction negative controls and these were

162 sequenced along with the sample libraries. Sequence reads from these controls were analysed 163 as reads from sample libraries (see below) and yielded less than $0.0094 \%$ and $0.016 \%$ of

164 reads mapping back to the genomes of horse and cod, respectively.

165

166 Analyses

167 Sequence reads were processed using the PALEOMIX pipeline v. 1.2.4 (Schubert, et al. 168 2014). Adapters were removed and reads were collapsed and trimmed as described in 169 (Orlando, et al. 2013) using AdapterRemoval version 2.1.7, discarding collapsed reads below $17025 \mathrm{bp}$ in length (Schubert, et al. 2016). Collapsed reads were down sampled to the lowest 171 number of reads $(894,682)$ and mapped to EquCab2.0 (Wade, et al. 2009) and GadMor2

172 (Tørresen, et al. 2016) excluding the mitochondrial genome, using the backtrack algorithm 173 from BWA v.0.5.10 ( $\mathrm{Li}$ and Durbin 2009) with seeding disabled as implemented in 174 PALEOMIX. Sequence reads were considered endogenous if they aligned to their respective 175 reference genome with a minimum alignment quality (MinQ value) of 25. Duplicates were 176 removed and a variety of summary statistics including endogenous DNA content, clonality, 177 average read length and GC-content was directly provided by PALEOMIX. Statistical tests 178 were applied when comparing treatments (paired t-test, or Wilcoxon Signed Rank if normality 179 is rejected using the Shapiro-Wilk normality test (Shapiro and Wilk 1965)). Critical 180 significance levels were adjusted using Bonferroni's correction for the number of multiple 181 tests in this study.

182 We estimated library complexity in sequence data obtained from three treatments 183 (DD, BleDD, BleDD ${ }^{2}$ ). Library complexity predictions from small datasets of shallow 184 sequencing can give false estimates of library complexity (Daley and Smith 2013). We 
185 therefore down sampled the collapsed sequencing files of each sample-treatment combination

186 (using seqtk, https://github.com/lh3/seqtk), matching the lowest number of sequences per

187 individual sample, instead of matching the lowest number in the entire dataset (as above), to

188 allow for a direct comparison of sequencing efficiency (defined as the fraction of non-clonal

189 endogenous DNA sequences divided by total sequences) using as much data as possible.

190 Several signatures of DNA damage were explored to investigate if any of the

191 treatments result in compositional bias or introduced further damage to the DNA. We

192 obtained read-length distributions, aDNA damage patterns and calculated the probability of

193 observing cytosine deamination in double strand context $(\delta d)$, cytosine deamination in single

194 strand context $(\delta s)$, and the probability of terminating in a single-stranded overhang $(\lambda)$ using

195 mapDamage v.2.0.6 (Ginolhac, et al. 2011; Jonsson, et al. 2013). Atlantic cod and horse

196 alignment files were down sampled to $1,000,000$ and 500,000 reads, respectively, when more

197 reads were sequenced.

\section{Microbial analyses}

199 We investigated the effect of the different pre-extraction treatments on the microbial

200 composition in the read data obtained from Atlantic cod and horses. We calculated relative

201 abundances by running the microbial profiling pipeline metaBIT (Louvel, et al. 2016),

202 aligning collapsed reads using Bowtie2 v2.1.0 (Langmead and Salzberg 2012) to the

203 MetaPhlAn2 database (version 2.0, excluding eukaryotes and viruses Truong, et al. 2015) and

204 excluding PCR duplicates with a modified version of the FilterUniqueBAM Python script

205 from PALEOMIX (Schubert, et al. 2014). Microbial profiles were generated at all

206 taxonomical levels from kingdom to strain using MetaPhlAn (Segata, et al. 2012; Truong, et

207 al. 2015), excluding low-abundance taxa $(<1 \%)$ to reduce statistical noise due to false

208 positives. These profiles were compared to 689 profiles from the Human Microbiome Project 
209 (Consortium 2012) and 15 profiles from soil samples (Fierer, et al. 2012; Louvel, et al. 2016)

210 to detect possible human microbial contamination from handling of the specimens and the 211 depositional context using Principal Coordinate Analyses (PCoA) of Bray-Curtis distances with 212 the R function pcoa. We also compared microbial profiles by hierarchical clustering with an 213 average linkage clustering method and 10,000 bootstrap iterations using the R package pvclust 214 (Suzuki and Shimodaira 2006) adapted for Bray-Curtis distances in metaBIT (Louvel, et al. 215 2016).

216 


\section{Results}

218 For each DNA extraction -following a short pre-digestion (SD), a double digestion (DD), 219 bleach wash followed by a short pre-digestion (BleSD) or a double digestion (BleDD), or 220 BleDD with twice the amount of starting material $\left(\mathrm{BleDD}^{2}\right)$ - between 1 and 36 million 221 collapsed reads were obtained (Table S2, Supporting Information). Washing bone powder 222 with bleach (comparing DD v BleDD) significantly (paired $\mathrm{t}=-4.095, \mathrm{DF}=11, p=0.0026$, 223 Fig. 2) increased the proportion of high quality and uniquely mapped endogenous reads, after 224 correcting for multiple significance tests $(n=15$, corrected $p$-value $=0.0033)$. BleDD 225 treatment increased the proportion of endogenous DNA by 1.3 to 3-fold relative to DD in all 226 but one of the samples (sample COD034, Fig. 3). No significant difference in clonality 227 (Wilcoxon Signed Rank, $W=29, N=12, p>0.05$; Fig. 2) or \%GC was observed between $228 \mathrm{DD}$ and BleDD (paired $\mathrm{t}=2.172, \mathrm{DF}=11, p=0.054$; Fig. S1, Supporting Information). 229 Unsurprisingly, libraries created after doubling the amount of starting material (BleDD ${ }^{2}$ ) 230 needed fewer amplification cycles, consistent with the increased amount of templates 231 available. These libraries showed reduced clonality levels and a further increase in the 232 proportion of high-quality and uniquely mapping endogenous DNA. Library efficiency shows 233 an average $1.8(\mathrm{BleDD})$ to $2.5\left(\mathrm{BleDD}^{2}\right)$ fold enrichment compared to DD (Fig. 3), when 234 measured as the fraction of high-quality and uniquely mapping reads obtained after down 235 sampling to match the lowest number of collapsed reads per bone.

236 The Atlantic cod libraries created after a short digestion (SD and BleSD) had 237 substantially lower endogenous content relative to their respective double digest treatments 238 (DD, BleDD or BleDD², Fig. 2). These libraries also differed in several other characteristics. 239 The mean \%GC of both short digestion treatments (SD and BleSD) was significantly higher 240 (paired $\mathrm{t}=6.449, \mathrm{DF}=4, p=0.0031$, Fig. S3, Supporting Information) compared to the 
241 double digestion treatments (DD, BleDD and BleDD ${ }^{2}$ ). Furthermore, the mean length of the

242 endogenous DNA reads was significantly lower in the short digestion treatments (SD and

243 BleSD) than those from DD, BleDD and $\mathrm{BleDD}^{2}$ (paired $\mathrm{t}=-16.56, \mathrm{DF}=4, p=7.78 \mathrm{E}-5$, Fig.

244 4). It is noteworthy that the distinct $\sim 10$ bp periodic peaks in length that are associated with

245 nucleosome protection (Pedersen, et al. 2014) were not present in both SD and BleSD

246 libraries in most cod samples (with the exception of the BleSD treatment in COD027), but

247 were present in all libraries after double digestion (with or without bleach). Such periodic

248 profiles were not found in all horse samples (Fig. 4), likely reflecting different DNA

249 preservation conditions. Mean length of endogenous DNA was not significantly different

250 between DD and BleDD for the Atlantic cod and horse samples combined (paired $\mathrm{t}=-2.36$,

$251 \mathrm{DF}=11, p=0.039$, which is not significant after Bonferroni correction).

252 All aDNA libraries showed the typical aDNA fragmentation and mis-incorporation

253 patterns, regardless of treatment (see COD029 as a representative example in Fig. S2,

254 Supporting Information). The mean probability of cytosine deamination in double strand

255 context $(\delta d)$, cytosine deamination in single strand context $(\delta s)$, and the mean probability of

256 terminating in an overhang $(\lambda)$ did not differ significantly after bleach treatment in the

257 Atlantic cod and horse samples (Table S3, Supporting Information) and bleach did not

258 consistently lead to damage probabilities for $\delta d, \delta s$ and $\lambda$ outside of the range observed in

259 non-bleach treatments (see also Fig. S3 and Fig. S4, Supporting Information for the 95\%

260 confidence intervals for simulated posterior distributions of $\delta d$, $\delta s$ and $\lambda$ per sample and

261 treatment).

262

263

Microbial analyses 
264 The mean abundance of reads that are classified as microbial using metaBIT did not differ 265 significantly between DD and BleDD (Wilcoxon Signed Rank, $W=19, N=12, p>0.05$ ) or 266 between short digestion (SD and BleSD) or double digestion treatments (DD, BleDD and 267 BleDD ${ }^{2}$; paired $\mathrm{t}=0.93, \mathrm{DF}=4, p=1$, Fig. 4). In contrast, the mean abundance of microbial 268 reads was significantly higher for Atlantic cod compared to horse $(\mathrm{t}=-9.05, \mathrm{DF}=10, p=$ 269 9.28E-05, Fig. 5). The microbial profiles generated from the ancient cod and horse samples 270 fall outside the diversity of human microbiomes and cluster closely to those obtained from 271 soil (Fig. S5, Supporting Information). Nonetheless, we obtained no support for segregation 272 of microbial profiles by sample, species (cod or horse), or pre-extraction treatment type 273 following hierarchical clustering, with all bootstrap support below 63\% (Fig. S6, Supporting 274 Information). The most abundant microbial species belonged to the genera Streposporangium, 275 Pseudomonas, Brevibacterium and Sinobacterium (Fig. S7, Supporting Information). Profiles 276 from Atlantic cod contained several less abundant genera (e.g., Marinobacter, Halomonas 277 and Idiomarina) that have been associated with the marine environment (Fig. S7, Supporting 278 Information). 


\section{Discussion}

281 In this study, we found that washing ancient bone powder with a combined treatment of 282 bleach and short pre-digestion provides a simple, cost-effective approach that significantly

283 improves the accessible endogenous DNA content of ancient samples. The obvious risk of 284 such treatments lies in the potential loss of endogenous DNA during these washing steps, 285 which we tracked here by sequencing the removed supernatant (SD and BleSD). Our analyses 286 showed that the gains realized by removing a larger proportion of exogenous contaminants 287 relative to endogenous DNA far exceeds the loss of endogenous DNA. Indeed, we observed 288 that the proportion of accessible unique reads after bleach treatment increased in all samples, 289 signifying that the short bleach treatment did not compromise complexity among the template 290 molecules. In particular for the BleDD ${ }^{2}$ treatment of the Atlantic cod samples -whereby we 291 used twice the amount of input material with an aim to augment template availability and, 292 thus, the library complexity- gains in efficiency were on average 2.5 fold higher relative to 293 the DD treatment. These improvements appear to be multiplicative to the earlier recorded 294 improvements arising from the use of a pre-digestion approach alone (Der Sarkissian, et al. 295 2014; Gamba, et al. 2014; Damgaard, et al. 2015; Gamba, et al. 2016).

296 There have been conflicting observations regarding the potential of bleach to cause 297 DNA damage during treatment. While it has been suggested that bleach could introduce DNA 298 damage similar to those accumulated in aDNA templates (Garcia-Garcera, et al. 2011), this 299 effect has not been replicated in other studies (e.g. Kemp and Smith 2005; Korlevic, et al. 300 2015). Here too, we obtained no evidence for increased or altered fragmentation processes; no 301 consistent increases in cytosine deamination rates following bleach washing; and no altered $302 \% \mathrm{GC}$ after bleach treatment. Overall, sequence bias due to bleach treatment was negligible, 
303 and sequence data obtained after such treatment does not require specific modifications in 304 post-sequencing analyses.

305 We observed that the length distribution of endogenous DNA reads obtained for 306 Atlantic cod after the short digestion treatment (SD and BleSD) was significantly shorter, and 307 contained higher $\% \mathrm{GC}$ than those obtained after the double digest (DD, BleDD, BleDD ${ }^{2}$ ), 308 with little effect of bleach. Moreover, we found that the distinct patterns of a $\sim 10$ bp read309 length periodicity -which are associated with nucleosome protection (Pedersen, et al. 2014)310 were more apparent after double digestion. Our results show that DD treatment is associated 311 with longer, more complex aDNA templates that in Atlantic cod are also more profoundly 312 associated with nucleosome protection. Interestingly, our results indicate that treating samples 313 with a double digest could increase rather than decrease the potential to obtain epigenetic 314 information from aDNA sequence data, and that bleach does not hinder such applications.

315 It is hypothesized that (microbial) surface contaminants are more loosely bound to the 316 substrate and released first by bleach or a short pre-digestion step, leaving a higher proportion 317 of endogenous DNA (Korlevic, et al. 2015). This is consistent with the finding that EDTA 318 supernatants from ancient bone/tooth extracts are enriched in the pathogen Yersinia pestis 319 DNA compared to pellets (Schuenemann, et al. 2011). Nonetheless, we did not observe a 320 significant difference in the number of reads that can be classified as microbial after bleach or 321 double digest treatments, nor a significantly different microbial profile between treatments.

322 These latter results agree with the similarity in microbial profiles obtained from ancient horse 323 bones that were extracted after first and second digestion (Der Sarkissian, et al. 2014). We did 324 find a significantly higher abundance of microbial reads in Atlantic cod bones compared to 325 those from horse. While differences in preservation conditions cannot be excluded at this 326 stage, it is possible that the high porosity of fish bones presents a greater potential for 
327 microbial colonization post-mortem. Overall, the absence of a clear treatment effect on the

328 microbial abundance and profile suggests that the fold-increase in authentic DNA after a 329 bleach and short digestion treatment is not necessarily related to the (partial) removal of 330 specific microbial contaminants.

331 The microbial profiles generated from the ancient cod and horse samples cluster 332 closely to those obtained from soil, indicating that microbial contamination from humans has 333 had limited impact on our data. Indeed, the most abundant microbial orders we observed in 334 our samples have been previously associated with bones obtained from archaeological 335 excavations (Der Sarkissian, et al. 2014) and likely originate from the depositional context 336 from which these bones were retrieved. Nonetheless, some of the less abundant orders (e.g., 337 Marinobacter, Halomonas and Idiomarina) have been associated with the marine 338 environment and may also be partly associated with the host-microbiome of Atlantic cod 339 (Star, et al. 2013) rather than the depositional context. The majority of reads in our DNA 340 extracts remain unclassified, however, providing no further information regarding their 341 biological origins and source.

342 In summary, we present a simple and cost-effective protocol to increase the 343 endogenous DNA content of ancient bone. By combining two well-established methods, we 344 obtained a substantial increase in the endogenous DNA content, greatly enhancing the 345 feasibility of recovering genome-scale data in such samples.

\section{Acknowledgements}

348 We are grateful to Anne-Karin Hufthammer and Jan Bill for the horse bone from the Gokstad 349 ship burial, and we thank Vida Rajkovaca for helping with the identification of the horse 350 bones from Orkney. This work was supported by the Research Council of Norway projects 
“Tracking Viking-assisted dispersal using ancient DNA (\#230821/F20 to SB), "Fisheries induced evolution in Atlantic cod investigated by ancient and historic samples" (\#203850/E40), “The Aqua Genome Project” (\#221734/O30), the Leverhulme Trust (to JB), the Danish Council for Independent Research, Natural Sciences (\#4002-00152B) and Initiative d'Excellence Chaires d'attractivité, Université de Toulouse (OURASI to LO).

\section{References}

Barrett JH. 2012. Being an Islander: Production and Identity at Quoygrew, Orkney, AD 9001600: McDonald Institute for Archaeological Research.

Barrett JH, Slater A. 2009. New excavations at the Brough of Deerness: power and religion in Viking Age Scotland. Journal of the North Atlantic 2:81-94.

Bonde N, Christensen AE. 1993. Dendrochronological dating of the Viking Age ship burials at Oseberg, Gokstad and Tune, Norway. Antiquity 67:575-583.

Briggs AW, Good JM, Green RE, Krause J, Maricic T, Stenzel U, Lalueza-Fox C, Rudan P, Brajkovic D, Kucan Z, et al. 2009. Targeted Retrieval and Analysis of Five Neandertal mtDNA Genomes. Science 325:318-321.

Campos PF, Craig OE, Turner-Walker G, Peacock E, Willerslev E, Gilbert MTP. 2012. DNA in ancient bone-Where is it located and how should we extract it? Annals of AnatomyAnatomischer Anzeiger 194:7-16.

Carpenter ML, Buenrostro JD, Valdiosera C, Schroeder H, Allentoft ME, Sikora M, Rasmussen M, Gravel S, Guillén S, Nekhrizov G. 2013. Pulling out the 1\%: wholegenome capture for the targeted enrichment of ancient DNA sequencing libraries. The American Journal of Human Genetics 93:852-864.

Castellano S, Parra G, Sánchez-Quinto FA, Racimo F, Kuhlwilm M, Kircher M, Sawyer S, Fu Q, Heinze A, Nickel B. 2014. Patterns of coding variation in the complete exomes of three Neandertals. Proceedings of the National Academy of Sciences 111:6666-6671.

Consortium HMP. 2012. Structure, function and diversity of the healthy human microbiome. Nature 486:207-214.

Daley T, Smith AD. 2013. Predicting the molecular complexity of sequencing libraries. Nature Methods 10:325-327.

Damgaard PB, Margaryan A, Schroeder H, Orlando L, Willerslev E, Allentoft ME. 2015. Improving access to endogenous DNA in ancient bones and teeth. Scientific reports 5.

Der Sarkissian C, Allentoft ME, Ávila-Arcos MC, Barnett R, Campos PF, Cappellini E, Ermini L, Fernández R, da Fonseca R, Ginolhac A. 2015. Ancient genomics. Philosophical Transactions of the Royal Society of London B: Biological Sciences 370:20130387.

Der Sarkissian C, Ermini L, Jonsson H, Alekseev AN, Crubezy E, Shapiro B, Orlando L. 2014. Shotgun microbial profiling of fossil remains. Molecular Ecology 23:1780-1798.

Enk JM, Devault AM, Kuch M, Murgha YE, Rouillard J-M, Poinar HN. 2014. Ancient whole genome enrichment using baits built from modern DNA. Molecular Biology and Evolution 31:1292-1294. 
Fierer N, Leff JW, Adams BJ, Nielsen UN, Bates ST, Lauber CL, Owens S, Gilbert JA, Wall DH, Caporaso JG. 2012. Cross-biome metagenomic analyses of soil microbial communities and their functional attributes. Proceedings of the National Academy of Sciences 109:21390-21395.

Gamba C, Hanghoj K, Gaunitz C, Alfarhan AH, Alquraishi SA, Al-Rasheid KA, Bradley DG, Orlando L. 2016. Comparing the performance of three ancient DNA extraction methods for high-throughput sequencing. Mol Ecol Resour 16:459-469.

Gamba C, Jones ER, Teasdale MD, McLaughlin RL, Gonzalez-Fortes G, Mattiangeli V, Domboróczki L, Kővári I, Pap I, Anders A, et al. 2014. Genome flux and stasis in a five millennium transect of European prehistory. Nat Commun 5.

Garcia-Garcera M, Gigli E, Sanchez-Quinto F, Ramirez O, Calafell F, Civit S, Lalueza-Fox C. 2011. Fragmentation of contaminant and endogenous DNA in ancient samples determined by shotgun sequencing; prospects for human palaeogenomics. Plos One 6:e24161.

Gilbert M, Bandelt H-J, Hofreiter M, Barnes I. 2005. Assessing ancient DNA studies. Trends Ecol Evol 20:541 - 544.

Ginolhac A, Rasmussen M, Gilbert MTP, Willerslev E, Orlando L. 2011. mapDamage: testing for damage patterns in ancient DNA sequences. Bioinformatics 27:2153-2155.

Ginolhac A, Vilstrup J, Stenderup J, Rasmussen M, Stiller M, Shapiro B, Zazula G, Froese D, Steinmann KE, Thompson JF. 2012. Improving the performance of true single molecule sequencing for ancient DNA. BMC Genomics 13:1.

Hofreiter M, Paijmans JL, Goodchild H, Speller CF, Barlow A, Fortes GG, Thomas JA, Ludwig A, Collins MJ. 2014. The future of ancient DNA: Technical advances and conceptual shifts. BioEssays.

Jonsson H, Ginolhac A, Schubert M, Johnson PLF, Orlando L. 2013. mapDamage2.0: fast approximate Bayesian estimates of ancient DNA damage parameters. Bioinformatics 29:1682-1684.

Kemp BM, Smith DG. 2005. Use of bleach to eliminate contaminating DNA from the surface of bones and teeth. Forensic science international 154:53-61.

Korlevic P, Gerber T, Gansauge M-T, Hajdinjak M, Nagel S, Ayinuer-Petri A, Meyer M. 2015. Reducing microbial and human contamination in DNA extractions from ancient bones and teeth. BioTechniques 59:87-93.

Langmead B, Salzberg SL. 2012. Fast gapped-read alignment with Bowtie 2. Nature Methods 9:357-359.

Lazaridis I, Patterson N, Mittnik A, Renaud G, Mallick S, Kirsanow K, Sudmant PH, Schraiber JG, Castellano S, Lipson M. 2014. Ancient human genomes suggest three ancestral populations for present-day Europeans. Nature 513:409-413.

Li H, Durbin R. 2009. Fast and accurate short read alignment with Burrows-Wheeler transform. Bioinformatics 25:1754-1760.

Li R, Liriano L. 2011. A bone sample cleaning method using trypsin for the isolation of DNA. Legal Medicine 13:304-308.

Louvel G, Der Sarkissian C, Hanghøj K, Orlando L. 2016. metaBIT, an integrative and automated metagenomic pipeline for analyzing microbial profiles from high - throughput sequencing shotgun data. Molecular ecology resources.

Malmström H, Svensson EM, Gilbert MTP, Willerslev E, Götherström A, Holmlund G. 2007. More on contamination: the use of asymmetric molecular behavior to identify authentic ancient human DNA. Molecular Biology and Evolution 24:998-1004. 
Meyer M, Kircher M. 2010. Illumina Sequencing Library Preparation for Highly Multiplexed Target Capture and Sequencing. Cold Spring Harbor Protocols 2010:pdb.prot5448.

Meyer M, Kircher M, Gansauge M-T, Li H, Racimo F, Mallick S, Schraiber JG, Jay F, Prüfer K, de Filippo C. 2012. A high-coverage genome sequence from an archaic Denisovan individual. Science 338:222-226.

Orlando L, Ginolhac A, Raghavan M, Vilstrup J, Rasmussen M, Magnussen K, Steinmann KE, Kapranov P, Thompson JF, Zazula G, et al. 2011. True single-molecule DNA sequencing of a pleistocene horse bone. Genome Research 21:1705-1719.

Orlando L, Ginolhac A, Zhang G, Froese D, Albrechtsen A, Stiller M, Schubert M, Cappellini E, Petersen B, Moltke I, et al. 2013. Recalibrating Equus evolution using the genome sequence of an early Middle Pleistocene horse. Nature 499:74-78.

Pedersen JS, Valen E, Velazquez AM, Parker BJ, Rasmussen M, Lindgreen S, Lilje B, Tobin DJ, Kelly TK, Vang S, et al. 2014. Genome-wide nucleosome map and cytosine methylation levels of an ancient human genome. Genome Res 24:454-466.

Pinhasi R, Fernandes D, Sirak K, Novak M, Connell S, Alpaslan-Roodenberg S, Gerritsen F, Moiseyev V, Gromov A, Raczky P. 2015. Optimal ancient DNA yields from the inner ear part of the human petrous bone. Plos One 10:e129102.

Poinar HN, Cooper A. 2000. Ancient DNA: do it right or not at all. Science 5482:1139.

Reich D, Green R, Kircher M, Krause J, Patterson N, Durand E, Viola B, Briggs A, Stenzel U, Johnson P, et al. 2010. Genetic history of an archaic hominin group from Denisova Cave in Siberia. Nature 468:1053 - 1060.

Rizzi E, Lari M, Gigli E, De Bellis G, Caramelli D. 2012. Ancient DNA studies: new perspectives on old samples. Genet Sel Evol 44:21.

Schroeder H, Ávila-Arcos MC, Malaspinas A-S, Poznik GD, Sandoval-Velasco M, Carpenter ML, Moreno-Mayar JV, Sikora M, Johnson PL, Allentoft ME. 2015. Genome-wide ancestry of 17th-century enslaved Africans from the Caribbean. Proceedings of the National Academy of Sciences 112:3669-3673.

Schubert M, Ermini L, Der Sarkissian C, Jónsson H, Ginolhac A, Schaefer R, Martin MD, Fernández R, Kircher M, McCue M. 2014. Characterization of ancient and modern genomes by SNP detection and phylogenomic and metagenomic analysis using PALEOMIX. Nature Protocols 9:1056-1082.

Schubert M, Lindgreen S, Orlando L. 2016. AdapterRemoval v2: rapid adapter trimming, identification, and read merging. BMC Research Notes 9:1.

Schuenemann VJ, Bos K, DeWitte S, Schmedes S, Jamieson J, Mittnik A, Forrest S, Coombes BK, Wood JW, Earn DJ. 2011. Targeted enrichment of ancient pathogens yielding the pPCP1 plasmid of Yersinia pestis from victims of the Black Death. Proceedings of the National Academy of Sciences 108:E746-E752.

Segata N, Waldron L, Ballarini A, Narasimhan V, Jousson O, Huttenhower C. 2012. Metagenomic microbial community profiling using unique clade-specific marker genes. Nature Methods 9:811-814.

Shapiro SS, Wilk MB. 1965. An analysis of variance test for normality (complete samples). Biometrika 52:591-611.

Star B, Haverkamp TH, Jentoft S, Jakobsen KS. 2013. Next generation sequencing shows high variation of the intestinal microbial species composition in Atlantic cod caught at a single location. BMC microbiology 13:1.

Suzuki R, Shimodaira H. 2006. Pvclust: an R package for assessing the uncertainty in hierarchical clustering. Bioinformatics 22:1540-1542. 
498

499

500

501

502

503 from all authors.

\section{Data accessibility}

Tørresen OK, Star B, Jentoft S, Reinar WB, Grove H, Miller JR, Walenz BP, Knight J, Ekholm JM, Peluso P, et al. 2016. An improved genome assembly uncovers a prolific tandem repeat structure in Atlantic cod. bioRxiv.

Truong DT, Franzosa EA, Tickle TL, Scholz M, Weingart G, Pasolli E, Tett A, Huttenhower C, Segata N. 2015. MetaPhlAn2 for enhanced metagenomic taxonomic profiling. Nature Methods 12:902-903.

Wade C, Giulotto E, Sigurdsson S, Zoli M, Gnerre S, Imsland F, Lear T, Adelson D, Bailey E, Bellone R. 2009. Genome sequence, comparative analysis, and population genetics of the domestic horse. Science 326:865-867.

All individual read data are available at the European Nucleotide Archive (ENA, www.ebi.ac.uk/ena) under study accession number PRJEB15516.

\section{Author contributions}

SB and BS designed the study. SB, BS, KH and CDR performed the analyses. SB and BS interpreted the results with input from LO. HN and AG did the laboratory work. JHB led the excavation and identification of the Orkney samples. SB and BS wrote the paper with input

504

505 
507 Figure 1. Overview of the experimental design. Bone powder was divided into two aliquots 508 of which one is treated with $0.5 \%$ bleach for 15 minutes. After a short, one-hour predigestion, DNA was extracted from the supernatant (SD and BleSD). The pelleted bone powder was subjected to a second, overnight digestion (double digestion) using a fresh buffer, followed by DNA extraction (DD and BleDD). The protocol BleDD ${ }^{2}$ (not shown in figure) is identical to BleDD except that twice the amount of the starting material was used, divided over two aliquots (see material and methods for details). Photograph shows specimen COD028, which was used in this study.

Figure 2. Level of endogenous DNA and clonality Atlantic cod and horse after different preextraction treatments. DNA was extracted from ancient Atlantic cod (COD027-COD034) and horse (VHR015-VHR064) bones, after a short pre-digestion (SD, grey), bleach combined with the short pre-digestion (BleSD, orange), a double digestion (DD, dark grey), bleach combined with a double digestion (BleDD, red) and bleach combined with a double digestion with twice the amount of input material (BleDD ${ }^{2}$, dark red). For horse samples only DD and BleDD data were obtained. The observed clonality for BleDD of sample COD034 is an extreme outlier and not depicted to scale. Collapsed reads were down sampled to the lowest number obtained $(n=894682)$.

Figure 3. Relative increase in library efficiency after bleach treatment. The fold-increase in library efficiency for BleDD (orange) and BleDD ${ }^{2}$ (dark red) is calculated relative to the DD treatment (scaled at 1) for Atlantic cod (COD027-COD034) and horse (VHR015-VHR064). Library efficiency is measured as the fraction of high quality unique reads, after down sampling to match the lowest number of collapsed reads per bone. For horse samples only BleDD data were obtained. The dashed lines indicate the average increases for the respective treatments (BleDD and BleDD ${ }^{2}$ ).

Figure 4. Length distributions of endogenous DNA templates from ancient bones. Size distributions are obtained for Atlantic cod (a) and horse (b) using the fraction of collapsed paired-end sequencing reads. DNA was extracted after a short pre-digestion (SD, grey), a bleach wash combined with the short digestion (BleSD, orange), a double digestion (DD, dark-grey), a bleach wash combined with a double digestion (BleDD, red) and a bleach wash combined with a double digestion with twice the amount of input material (BleDD ${ }^{2}$, darkred).

Figure 5. Microbial content of 12 ancient Atlantic cod and horse bones after different preextraction treatments. DNA was extracted from ancient Atlantic cod (COD027-COD034) and horse (VHR015-VHR064) bones, after a short pre-digestion (SD, grey), bleach combined with the short pre-digestion (BleSD, orange), a double digestion (DD, dark grey), bleach combined with a double digestion (BleDD, red) and bleach combined with a double digestion with twice the amount of input material (BleDD ${ }^{2}$, dark red). For horse samples only DD and BleDD data were obtained. Reads were classified at the genus level with metaBIT, aligning reads to the MetaPhlAn2 database. 


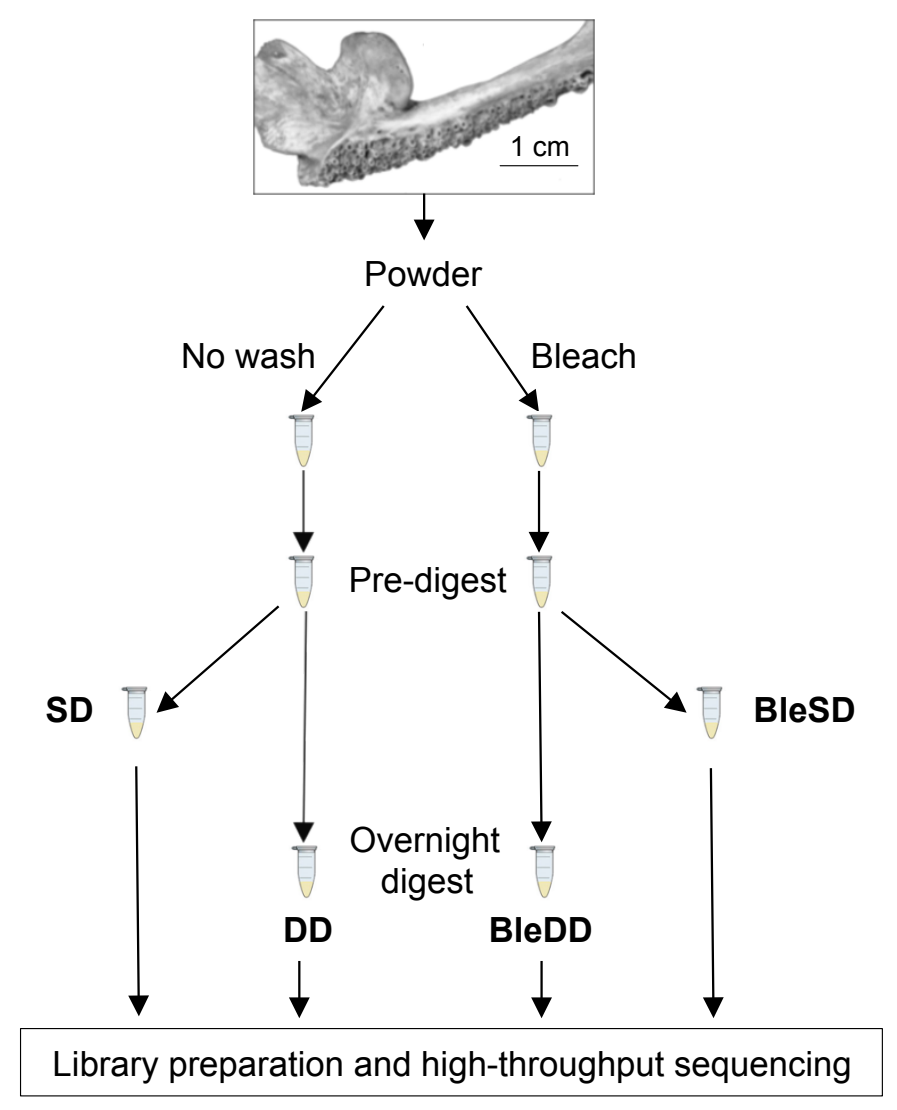




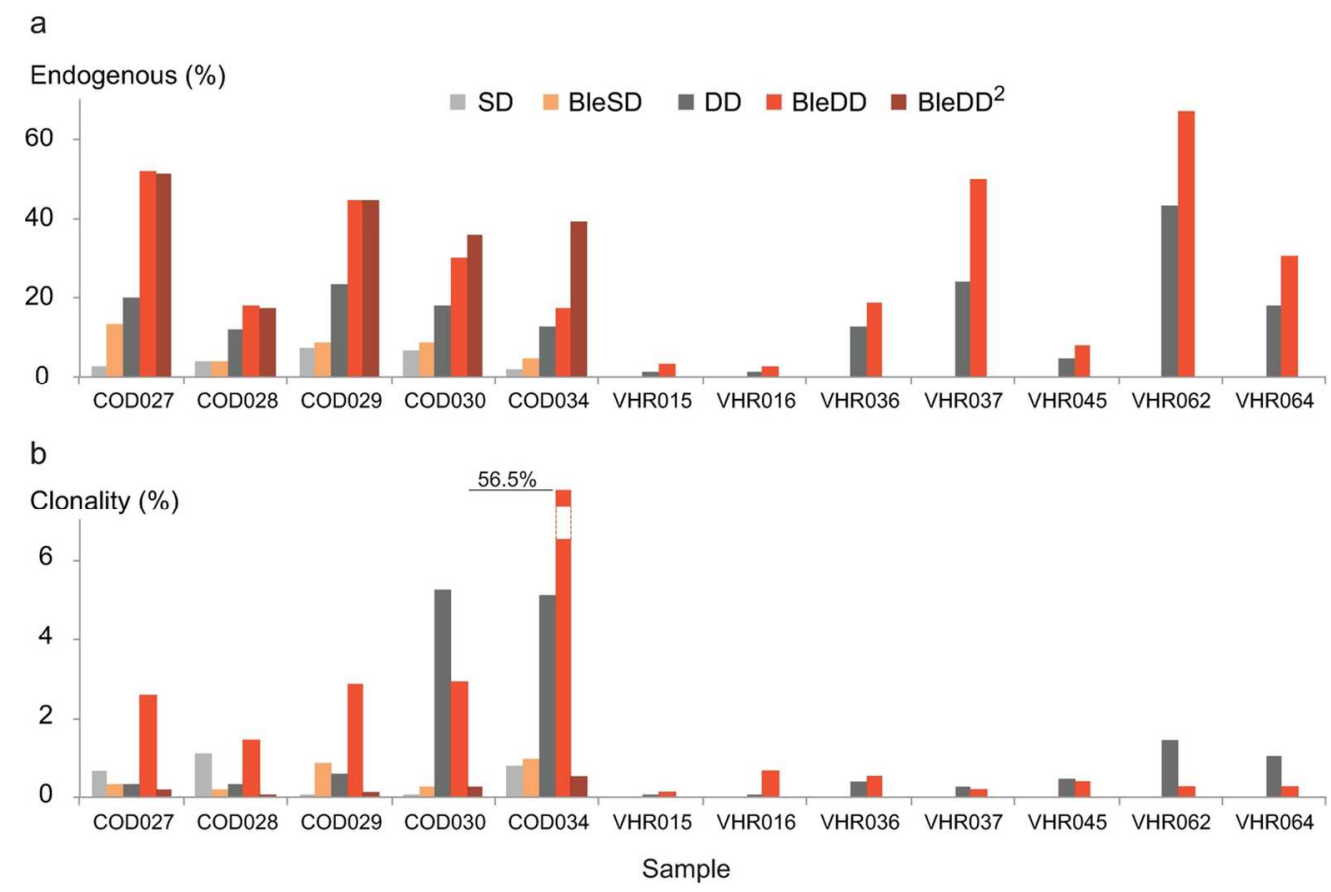

$118 \times 79 \mathrm{~mm}(300 \times 300 \mathrm{DPI})$ 


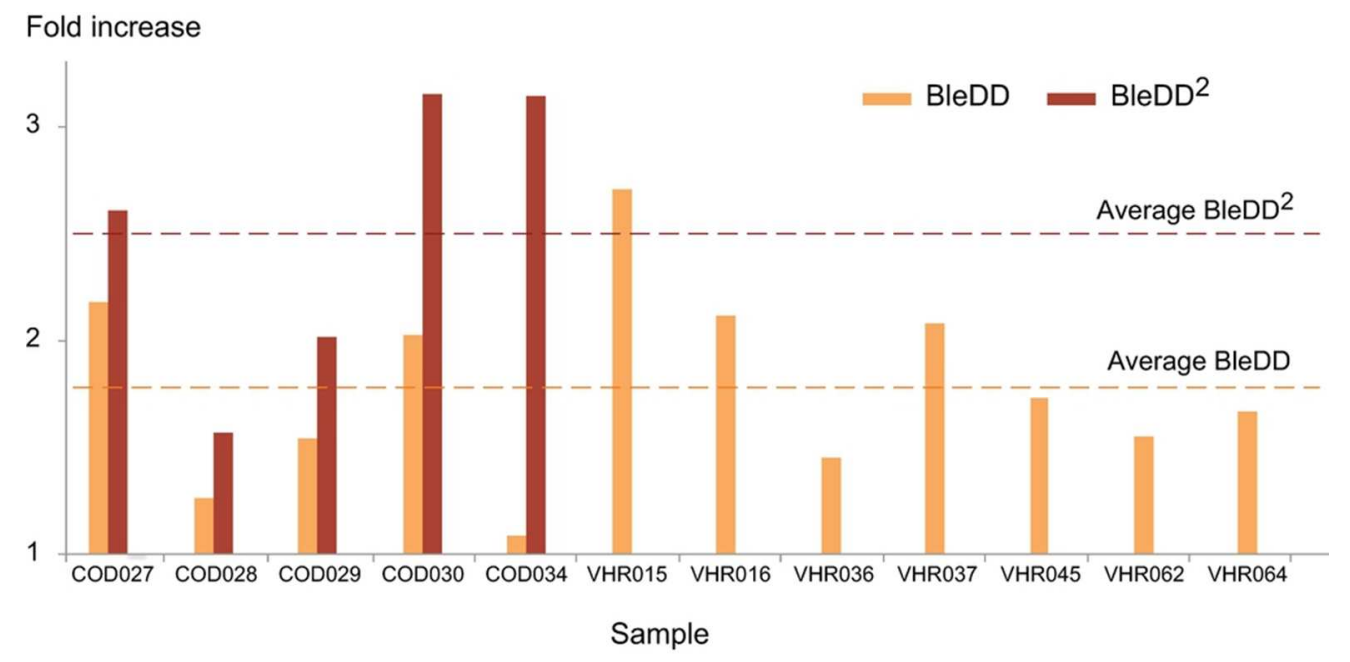

$85 \times 41 \mathrm{~mm}(300 \times 300$ DPI $)$ 
a

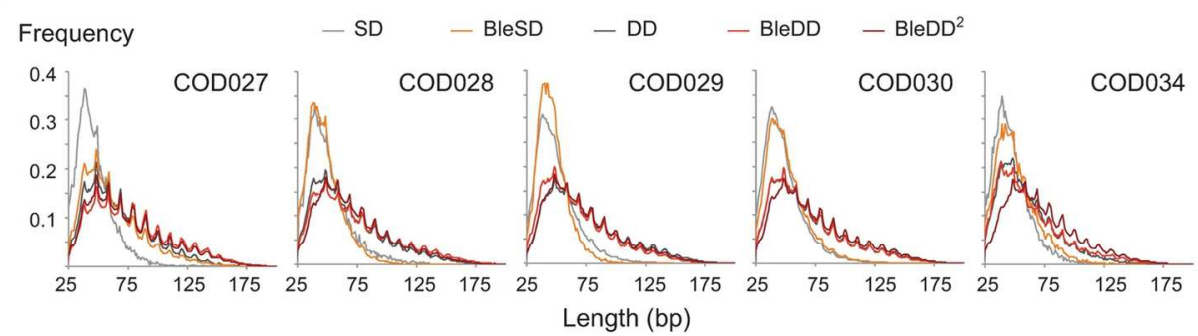

b
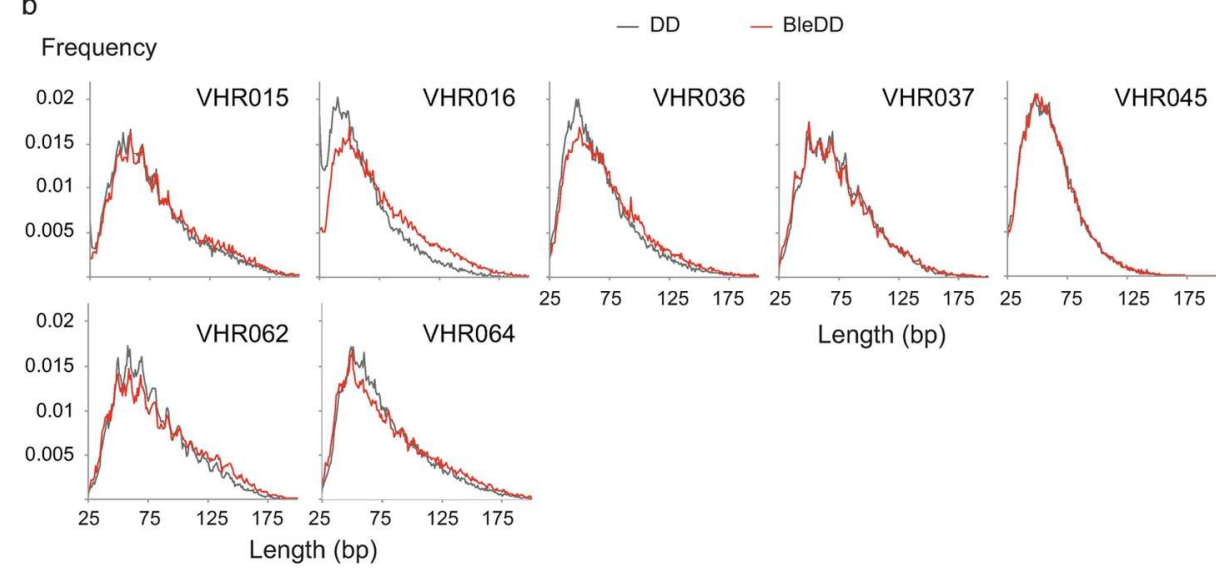

$138 \times 102 \mathrm{~mm}(300 \times 300$ DPI $)$ 


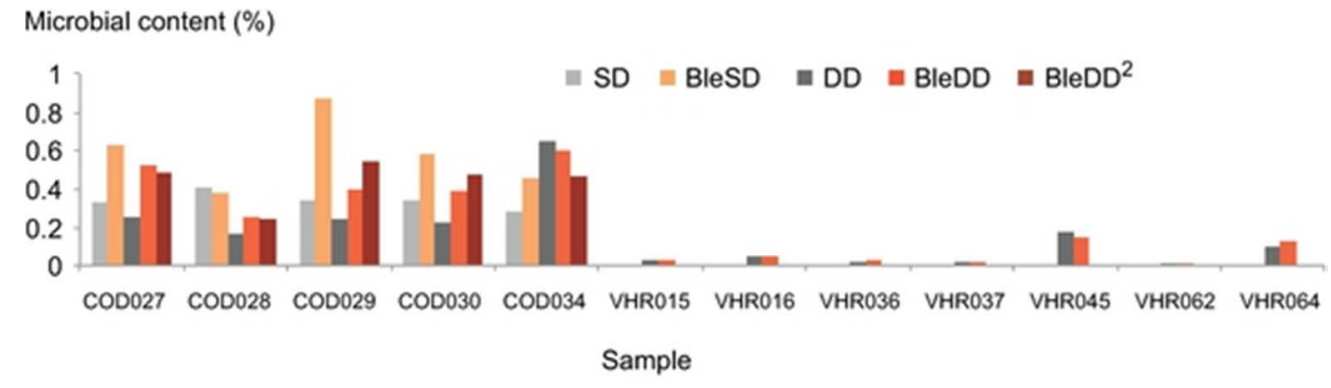

$49 \times 14 \mathrm{~mm}(300 \times 300 \mathrm{DPI})$ 